\title{
Design and Implementation of Vibration Isolation System for Mobile Doppler Wind LIDAR
}

\author{
Xiaoquan Song ${ }^{1 *}$, Chao Chen ${ }^{1,2}$, Bingyi Liu ${ }^{1}$, Jinbao Xia ${ }^{1}$, and Samo Stanič $\breve{c}^{3}$ \\ ${ }^{1}$ Ocean Remote Sensing Institute, Ocean University of China, 5 Yushan Road, Qingdao 266003, China \\ ${ }^{2}$ Shandong Provincial Key Laboratory of Ocean Environment Monitoring Technology, \\ Institute of Oceanographic Instrumentation, Shandong Academy of Sciences, \\ Qingdao, Shandong 266001, China \\ ${ }^{3}$ University of Nova Gorica, Vipavska 13, SI-5000 Nova Gorica, Slovenia
}

(Received November 5, 2012 : accepted December 17, 2012)

\begin{abstract}
The operation of a Doppler wind LIDAR in a mobile environment is very sensitive to shocks and vibrations, which can cause critical failures such as misalignment of the optical path and damage to optical components. To be able to stabilize the LIDAR and to perform wind field measurements in motion, a shock absorption and vibration isolation system was designed and implemented. The performance of the vehicle-mounted Doppler wind LIDAR was tested in motion, first in a circular test route with a diameter of about $30 \mathrm{~m}$ and later in regular expressway traffic. The vibration isolation efficiency of the system was found to be higher than $82 \%$ in the main vibration area and shock dynamic deflection was smaller than maximal deflection of the isolator. The stability of the laser locking frequency in the same mobile environment before and after the vibration isolation system installation was also found to be greatly improved. The reliability of the vibration isolation system was confirmed by good results of the analysis of the LIDAR data, in particular the plane position indicator of the line of sight velocity and the wind profile.
\end{abstract}

Keywords: Doppler wind LIDAR, Mobile measurements, Vibration isolation

OCIS codes : (280.3640) Lidar; (120.7280) Vibration analysis; (280.3340) Laser Doppler velocimetry

\section{INTRODUCTION}

Measurement of tropospheric winds has been recognized as one of the most important measurements for the atmospheric sciences. Global tropospheric wind measurements are routinely used for the improvement of large scale numerical weather prediction. High-accuracy measurements of the wind field on a limited scale can reveal local hydrodynamic properties such as streamlines and turbulences, which are essential for better understanding of mesoscale dynamic processes, air-mass transport and exchange of energy and matter with the surface. Modern remote sensing of wind fields in real time is becoming indispensable in a number of applications, such as airfield traffic control [1] and sailboat racing [2] where detailed knowledge of wind speed and directions provides increased safety or competitive advantage. One of the devices capable of these kinds of measurements is a Doppler wind LIDAR, which has so far proved to be a valuable tool in fixed location experiments. A number of applications would, however, require the operation of a Doppler wind LIDAR in a mobile environment, which has so far not been successful due to sensitivity to shock and vibrations, which resulted in critical failures such as misalignment of the optical path and damage to optical components. To stabilize a Doppler LIDAR system to the degree where it is possible to perform wind field measurements in motion, a shock absorption and vibration isolation system was designed and implemented.

In our study we used an incoherent vehicle-mounted Doppler wind LIDAR based on an Iodine filter at $532 \mathrm{~nm}$, developed for atmospheric wind field measurements at the Ocean Remote Sensing Institute, Ocean University of China. Excellent performance of wind field measurements by this

\footnotetext{
*Corresponding author: songxq@ouc.edu.cn

Color versions of one or more of the figures in this paper are available online.
} 
system has been confirmed up to the heights of $15 \mathrm{~km}$ in the night and $12 \mathrm{~km}$ in the day time [3]. The system has also shown good performance in measuring sea surface winds (SSW) in high spatial and temporal resolution [4]. All these measurements were performed with the LIDAR system stationary, level-adjusted and stable, as the exact direction of the laser beam was critical for its good performance in elevation-over-azimuth scans [5]. Without an appropriate vibration isolation system, shock and vibration from the vehicle engine rotation, pumping exhaust and uneven road surface affect the LIDAR directly and were found to be the cause for system failures such as optical path misalignment and optical component damage [6,7]. These failures severely limited LIDAR operation.

In order to ensure stationary LIDAR operation immediately after it is transported to the measurement site, and the mechanical stability of its optical system during LIDAR operation in motion, a shock absorption and vibration isolation system was designed and implemented. The ability of a Doppler wind LIDAR to perform wind field measurements in motion may enable its use in a number of new vehicle-mounted, airborne and shipborne applications.

\section{DESIGN OF THE VIBRATION ISOLATION SYSTEM}

The incoherent vehicle-mounted Doppler wind LIDAR consists of four main components: the subsystem for the transmission, for the reception and frequency discrimination, for the data acquisition and the subsystem for scanning control [8]. Optical components are installed on a doublelayer optical table, which is mounted on a robust aluminum frame to ensure the desired strength and rigidity of the entire optical system with a total weight of $374 \mathrm{~kg}$. The transmitting subsystem and the receiving telescope are mounted on its upper layer, while the rest of the receiving

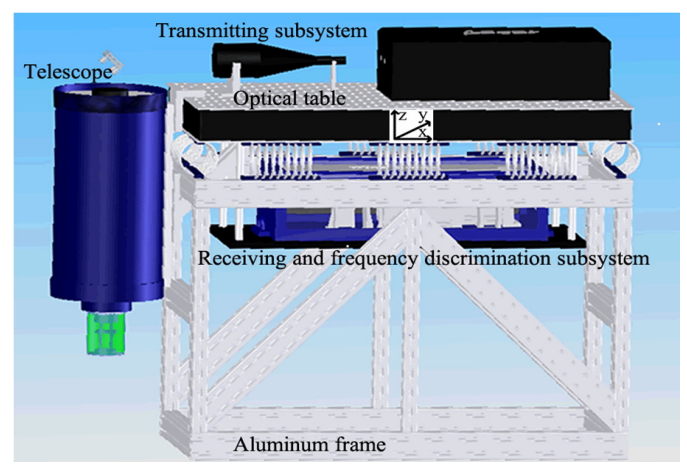

FIG. 1. Schematic view of the LIDAR system. The telescope and the subsystems for the transmission, reception and frequency discrimination are all mounted on the optical table, which is mechanically separated from the LIDAR frame and the vehicle by using a shock absorption and vibration isolation system. and frequency discrimination subsystem is mounted on its lower layer (Fig. 1).

The conceptual idea of the vibration isolation system design is to isolate the optical part from the vehicle and the rest of the LIDAR and to thus reduce the harmful influence of vibration on optical components. In our case, we used ten wire rope isolators (M12-425-08 by IDC Shock \& Vibration Isolation Specialists, USA), which have been proven in a variety of applications and performance tests [9]. The rigidity center of our vibration isolation system was designed to coincide with the center of gravity of the optical system and the isolating suspension elements were in the horizontal plane arranged uniformly and symmetrically around the center of gravity. This was shown to improve vibration isolation [10]. The isolators were installed between the upper optical table and the aluminum frame, each carrying equal vertical static loads of about $37.4 \mathrm{~kg}$, which was within their designed static load range [11].

The system was subsequently tested through the analysis of the vibration isolation efficiency, the anti-shock effect and the LIDAR data quality of the wind field measurements performed in motion. The frequency range of perturbations due to the interaction of the vehicle with the road surface was taken to be $5-500 \mathrm{~Hz}$, of perturbations due to automotive engine rotation $13-80 \mathrm{~Hz}$ and the total vibration frequency of a vehicle in motion was taken to be above $20 \mathrm{~Hz}$ [12].

The extent of vibration isolation can be expressed by transfer coefficient $T$. In the same excitation conditions, the smaller the isolation transfer coefficient is, the less force or displacement is passed through the isolation system, and the better the isolation effect is. $T$ is a function of the damping ratio $\xi$ and the frequency ratio $f / f_{n}$, where $f$ is the perturbation frequency and $f_{n}$ is the eigenfrequency of the system. The dependence of the transfer coefficient on the frequency ratio for different values of $\xi$ is shown in Fig. 2. The induced vibrations are being damped in the case when $T<1$, which corresponds to the frequency ratio $f / f_{n}$ $>\sqrt{2}$. The isolators thus play an important role in the design of vibration isolation, as they define the eigenfrequency and thus the frequency ratio. The isolators should not be too soft, as this results in larger system size and the isolation system is easier to shake, which affects the stability of the optical table [13]. In the design, dynamic parameters of the isolator candidates, such as dynamic stiffness coefficient, peak response frequency and vibration isolation efficiency were considered. For the selected M12-425-08 isolator the total vibration stiffness of the system is about $K_{V}=875 \mathrm{~N} / \mathrm{mm}$ and its eigenfrequency

$$
f_{n}=\frac{1}{2 \pi} \sqrt{\frac{K_{V}}{m}}
$$

was found to be about $7.7 \mathrm{~Hz}$. The peak response frequency $F_{n}$ of the optical system depends on the dynamic stiffness coefficient $\alpha$ as 


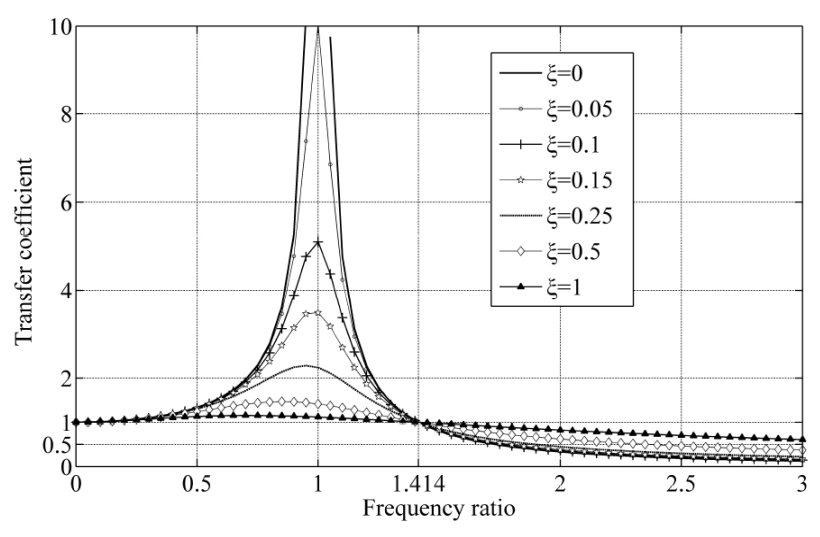

FIG. 2. The dependence of the transfer coefficient $T$ on the ratio between the stimulation frequency and the eigenfrequency of the system. The parameter $\xi$ indicates the damping ratio.

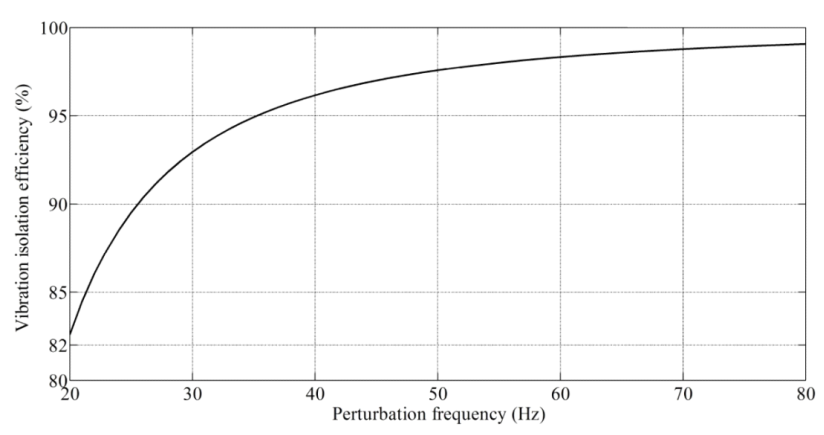

FIG. 3. The dependence of the vibration isolation efficiency $\varepsilon$ on the stimulation frequency $f$.

$$
F_{n}=f_{n} \sqrt{\alpha}
$$

For the coefficient $\alpha$, which generally ranges from 1.8 to 2.5 , the upper value yields peak response frequency of the optical system of about $12.2 \mathrm{~Hz}$. As this is considerably lower than the lowest perturbation frequency, resonance will not occur. The dependence of the isolation efficiency $\varepsilon$ on the perturbation frequency $f$,

$$
\varepsilon=1-\frac{1}{\left|1-\left(f / f_{n}\right)^{2}\right|},
$$

is graphically presented in Fig. 3. For perturbation frequencies above $20 \mathrm{~Hz}$ the efficiency $\varepsilon$ is always larger than 0.82 . For perturbations at lower frequencies, resonance may occur, but the damping ratio of the selected isolator is as high as 0.2 so the transfer coefficient will still be very low (Fig. 2). In total, the system is expected to provide good protection against vibrations in the entire applicable frequency range.

Short, abrupt perturbations (shocks) can also influence LIDAR measurements and cause serious damage to the optical components, so the response of the optical system to this kind of stimulations was also tested. Total shock stiffness $K_{S}$ of this vibration isolation system was found to be $499 \mathrm{~N} / \mathrm{mm}$. Based on the shock testing specifications [14], the mobile wind LIDAR was expected to be subjected to half sine shock pulses with peak acceleration $\alpha_{i}$ of 150 $\mathrm{m} / \mathrm{s}^{2}$, duration of $11 \mathrm{~ms}$ and repetition rate of about 45.5 Hz. According to the relations

$$
\begin{aligned}
& f_{s n}=\frac{1}{2 \pi} \sqrt{\frac{K_{s}}{m}}, \\
& \alpha_{0}=1.6 \alpha_{i} \frac{f_{s n}}{f_{i}} \text { and } \\
& \delta=\frac{m \alpha_{0}}{K_{S}},
\end{aligned}
$$

the eigenfrequency of the vibration isolation system to shock excitations $f_{s n}$ is about $5.8 \mathrm{~Hz}$, the response acceleration $\alpha_{0}$ is about $30.6 \mathrm{~m} / \mathrm{s}^{2}$ and the dynamic deflection $d$ is about $22.9 \mathrm{~mm}$, which is well below the maximum possible deflection of the vibration isolation system.

\section{THE EFFECT OF THE VIBRATION ISOLATION SYSTEM ON THE LOCKING FREQUENCY}

For successful operation of a Doppler wind LIDAR single longitudinal mode operation and frequency stability are essential. The pulse laser transmitter of our mobile Doppler wind LIDAR is excited by a dual wavelength seed injection laser and provides a tunable single longitudinal mode with the required frequency stability [15]. The stability of the laser frequency is obtained by locking the second harmonic of the seeded laser to the mid-point of a wing of Iodine

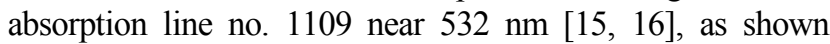
in Fig. 4. The vibration isolation system was required to preserve the frequency locking effect under vibration and

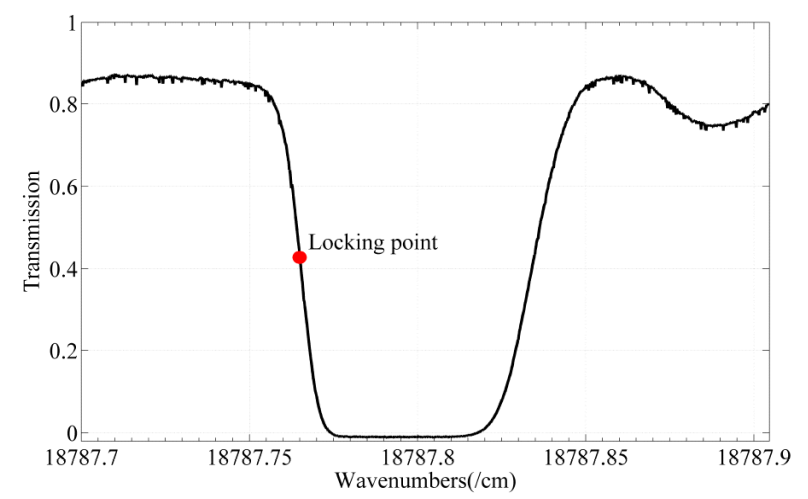

FIG. 4. The Iodine absorption line and the locking point of the seeded laser. 


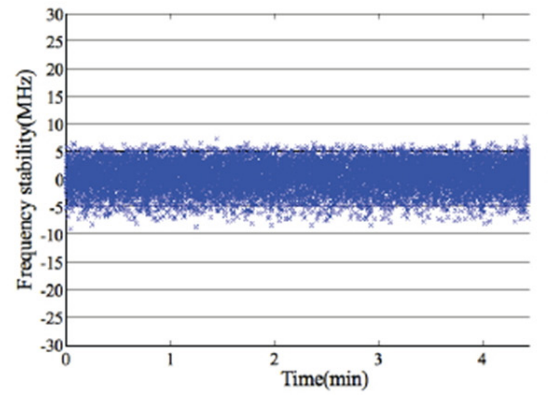

(a)

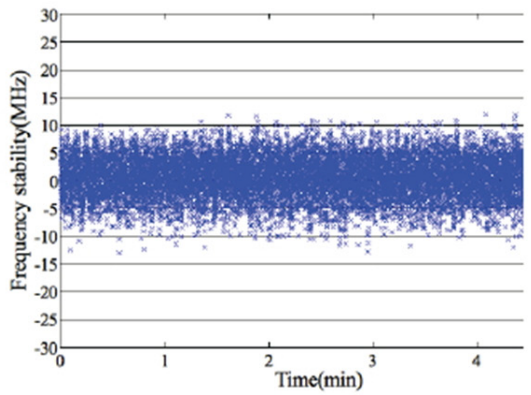

(b)

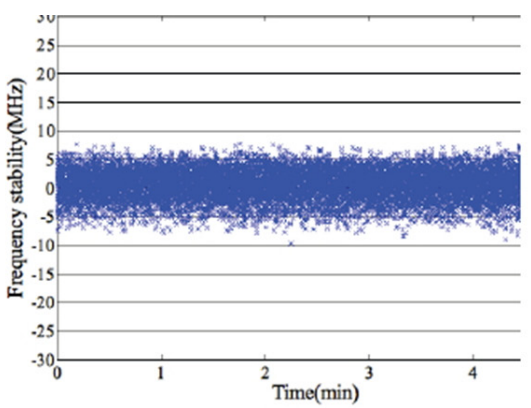

(c)

FIG. 5. Frequency locking of the laser in the case of no vibrations (a), $30 \mathrm{~Hz}$ from vehicle engine (b) and $30 \mathrm{~Hz}$ from vehicle engine with installed vibration isolation system (c).

was tested by simulated automotive engine excitations in a stationary environment. Comparisons of the locking frequency effect with and without the vibration isolation system are shown in Fig. 5. The standard deviation of the locking frequency uncertainty in a completely vibrationless environment was found to be $2.54 \mathrm{MHz}$ (Fig. 5, left). The vibration isolation system was found to considerably improve the frequency stability, as the uncertainty increased to $4.78 \mathrm{MHz}$ (Fig. 5, center) in the case of direct exposure to vibrations at 1800 rpm and only to $2.63 \mathrm{MHz}$ (Fig. 5, right) when the system was in the plane.

\section{WIND FIELD MEASUREMENTS IN MOTION}

With the vibration isolation system installed, we performed a number of measurements in motion, both along a circular test track and in real highway traffic conditions. As shown in Fig. 6, measurements performed during circular motion of the vehicle with fixed elevation and azimuth angles of the LIDAR are equivalent to stationary scanning measurements with the laser beam direction controlled by an elevation-over-azimuth scanner. In both cases, $V_{L O S}$ can be expressed as a function of the azimuth angle $\varphi$ as

$$
V_{L O S}(\varphi)=-V_{h} \cos \theta \cos (\beta-\varphi),
$$

where $V_{h}$ is the horizontal wind speed and $\theta$ the elevation of the LOS. Horizontal wind direction $\beta$ corresponds to the azimuth angle of the $V_{L O S}$ minimum. Test track measurements with the vibration isolation system installed were performed during circular movement along a $30 \mathrm{~m}$ diameter route on the campus of the Ocean University of China (Fig. 7, left) on 13 Dec 2009 between 18:07-18:26 coordinated universal time (UTC). From the measurements, wind profile was calculated based on the velocity-azimuth-display (VAD) method and under the assumption that wind speed and direction at fixed height were uniform and stable. The LOS velocity during circular motion at a given height during

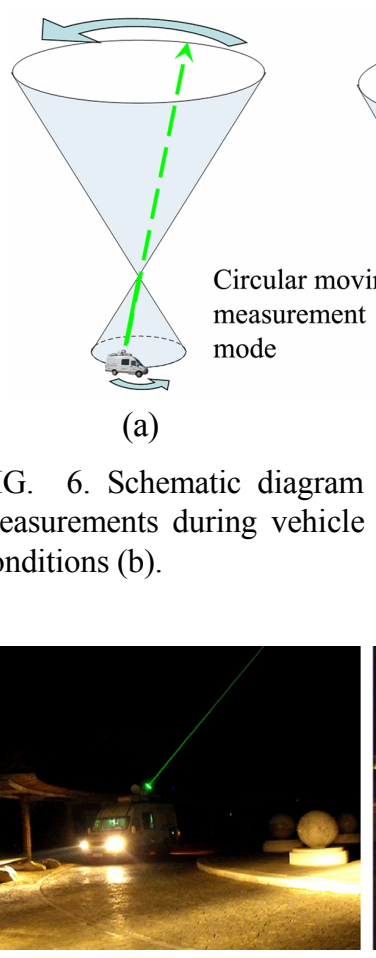

(a)

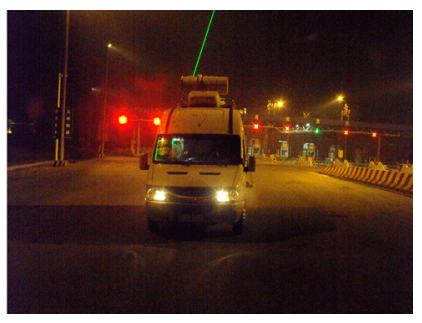

(b)
FIG. 7. Measurements during pre-defined circular vehicle motion on the campus of the Ocean University of China on 13 Dec 2009 between 18:07-18:26 UTC (a) and in regular expressway traffic conditions on G20 Expressway close to Qingdao, China, from 20:00-21:00 UTC on 18 December 2009 (b).

movement should be sinusoidal; the fits to LOS velocity at different altitudes then yield the wind direction and speed [5]. An example of the measured LOS velocity averaged over 9 loops with 45 deg elevation for the altitude of 0.5 $\mathrm{km}$ is shown in Fig. 8, where open squares represent data points and the full red line the cosine function fit. The retrieved values of the wind speed and horizontal wind direction ( $V_{h}$ and $\beta$ of Eq. 7), quality $\left(\chi^{2}\right)$ of the fit and variance $\left(\sigma^{2}\right)$ of the distribution of the data points with 


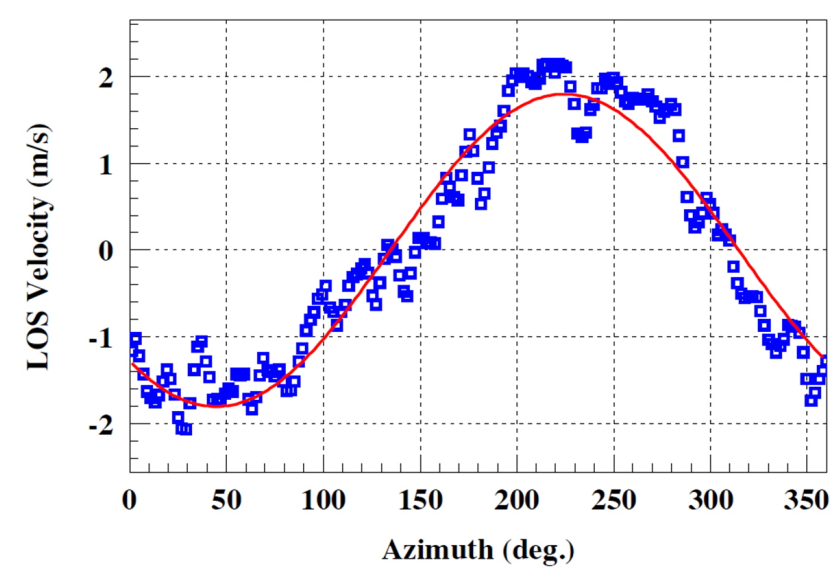

FIG. 8. An example of line of sight velocity at the height of 0.5 $\mathrm{km}$ above the LIDAR device during the measurement on 13 December 2009, 18:07-18:26 UTC. During the measurement, elevation and azimuth angles of the scanner were fixed and the vehicle was moving along a circular track. Data points are denoted by blue open squares and the fitted cosine function by the full red line.

TABLE 1 . Summary of the retrieved wind speed $V_{h}$, the azimuth angle $\beta$ of horizontal wind direction, the $\chi^{2}$ of the cosine fit and the variance $\sigma^{2}$ of the distribution of the $V_{L O S}$ data points with respect to the fitted cosine curve for four different heights. Uncertainties of $V_{h}$ and $\beta$ were obtained from the fit

\begin{tabular}{c|c|c|c|c}
\hline \hline $\begin{array}{c}\text { Height } \\
{[\mathrm{km}]}\end{array}$ & $\begin{array}{c}V_{h} \\
{[\mathrm{~m} / \mathrm{s}]}\end{array}$ & $\begin{array}{c}\beta \\
{[\mathrm{deg} .]}\end{array}$ & $\chi^{2}$ & $\begin{array}{c}\sigma^{2} \\
{\left[(\mathrm{~m} / \mathrm{s})^{2}\right]}\end{array}$ \\
\hline 0.5 & $2.54 \pm 0.09$ & $44.65 \pm 2.08$ & 0.29 & 0.39 \\
\hline 1.0 & $6.67 \pm 0.22$ & $19.68 \pm 1.89$ & 0.13 & 2.19 \\
\hline 1.5 & $2.52 \pm 0.24$ & $2.64 \pm 5.30$ & 0.91 & 2.61 \\
\hline 2.0 & $8.49 \pm 0.50$ & $5.10 \pm 3.14$ & 0.73 & 11.29 \\
\hline
\end{tabular}

respect to the fitted cosine curve are summarized in Table 1 for four different heights. Deviations of the data points from the expected cosine dependence in azimuth are due to irregularities in the circular shape of the track and wind fluctuations.

In addition to circular motion measurements the mobile Doppler LIDAR was tested in regular expressway traffic to confirm the stability of the LIDAR measurements in realistic conditions (Fig. 7, right). The data from azimuthal scanning was displayed real time on the wind LIDAR's data acquisition system and its performance was found to be good, as the vibration isolation system successfully reduced the induced the vibration and kept the optical system stable enough to be able to operate without any problems [17].

\section{CONCLUSION}

To conclude, we designed, constructed and implemented a vibration isolation system to protect the optical components of a mobile Doppler wind LIDAR from shocks and vibrations which arise when performing measurements in motion. In the tests, the system demonstrated high vibration isolation efficiency of more than $82 \%$ in the main vibration area and good anti-shock protection with shock dynamic deflection smaller than maximal deflection of the isolator. The implementation of the vibration isolation system also greatly improved the stability of the locking frequency of the seeded laser. Wind field parameters, including the planposition-indicator of the line-of-sight velocity and wind velocity and direction profiles were successfully obtained from LIDAR measurements in pre-determined circular motion as well as in regular expressway traffic. High quality of the obtained results encourages applications of our vibration isolation system to LIDARs at other mobile platforms, such as to ship-borne and airborne LIDARs.

\section{ACKNOWLEDGMENT}

This work was supported by the National Natural Science Foundation of China (projects no. 40905005 and 40906019) and by the State Key Laboratory of Severe Weather, Chinese Academy of Meteorological Sciences (project no. 2010LASWA07) and Institute of Urban Meteorology, CMA, China (project no. UMRF201002).

\section{REFERENCES}

1. C. M. Shun and P. W. Chan, "Applications of an infrared Doppler LIDAR in detection of wind shear," J. Atmos. Oceanic Technol. 25, 637-655 (2008).

2. G. L. Wang, L. P. Liu, Z. S. Liu, B. Lv, and R. Mu, "The application of sea-surface wind detection with Doppler LIDAR in Olympic sailing," Adv. Atmos. Sci. 28, 1471-1480 (2011).

3. Z. S. Liu, B. Y. Liu, Z. G. Li, Z. A. Yan, S. H. Wu, and Z. B. Sun, "Wind measurements with incoherent Doppler LIDAR based on iodine filters at night and day," Appl. Phys. B 88, 327-335 (2007).

4. Z. S. Liu, B. Y. Liu, S. H. Wu, Z. G. Li, and Z. J. Wang, "High spatial and temporal resolution mobile incoherent Doppler LIDAR for sea surface wind measurements," Opt. Lett. 33, 1485-1487 (2008).

5. B. Y. Liu, Z. S. Liu, X. Q. Song, S. H. Wu, D. C. Bi, X. T. Wang, Q. W. Yin, and O. Reitebuch, "Modifications and moving measurements of the mobile Doppler LIDAR," in Proc. of the Symposium Dragon 2 Programme Mid-term Results 2008-2010 (Guilin, P. R. China, May 2010), CD, SP-684, paper ID:5291.

6. V. S. Dmitriev, T. G. Kostyuchenko, V. S. Yangulov, and V. V. Teploukhov, "Mobile LIDARs. influence of external mechanical actions on accuracy of LIDAR aiming," Bull. Tomsk Polytech. Univ. 311, 28-31 (2007).

7. R. T. Menzies and D. M. Tratt, "Airborne $\mathrm{CO}_{2}$ coherent LIDAR for measurements of atmospheric aerosol and cloud backscatter," Appl. Opt. 33, 5698-5711 (1994). 
8. Z. S. Liu, Z. J. Wang, S. H. Wu, B. Y. Liu, Z. G. Li, X. Zhang, and D. C. Bi, "Fine-measuring technique and application for sea surface wind by mobile Doppler wind LIDAR," Opt. Eng. 48, 1 (2009).

9. A. M. Veprik, V. I. Babitsky, N. Pundak, and S. V. Riabzev, "Vibration protection of sensitive components of infrared equipment in harsh environments," Shock Vib. 8, 55-69 (2001).

10. E. Nakashio, S. Watanabe, S. Sasaki, Inaguma, Honda, Kurihara, Shishido, and T. Takahashi, "Vibration type gyro sensor," US Patent, US 2009/0320593 A1 (2009).

11. Isolator M12 Series Performance Data, http://www.isolator.com/ elecdocs $/ \mathrm{m} 12$ performancedata.pdf.

12. International Electrotechnical Commission, "IEC 60068-2-64 environmental testing, tests - test Fh: vibration, broadband random and guidance," http://webstore.iec.ch/ (2008).

13. Z. H. Gan, "Design of inner frame vibration absorbing system for optoelectronic pod," Opt. Precision Eng. (in Chinese) 9, 2036-2043 (2010).

14. International Electrotechnical Commission, "IEC 60068-2-27 environmental testing, tests - test Ea and guidance, shock," http://webstore.iec.ch/ (2008).

15. Z. S. Liu, D. Wu, J. T. Liu, K. L. Zhang, W. B. Chen, X. Q. Song, J. W. Hair, and C. Y. She, "Low-altitude atmospheric wind measurement from the combined Mie and Rayleigh backscattering by Doppler LIDAR with an Iodine filter," Appl. Opt. 41, 7079-7086 (2002).

16. Z. S. Liu, S. H. Wu, and B. Y. Liu, "Seed injection and frequency locked Nd:YAG laser for direct-detection wind LIDAR,” Opt. Laser Technol. 39, 541-545 (2007).

17. B. Y. Liu, Z. S. Liu, X. Q. Song, S. H. Wu, D. C. Bi, X. T. Wang, Q. W. Yin, and Y. Chen, "Mobile Doppler LIDAR with inertial navigation system for moving measurements," in Proc. of the 25th International Laser Radar Conference (St. Petersburg, Russia, Jul. 2010), CD, paper S01P-13. 